\title{
Thin elevation: A technique for achieving thin perforator flaps
}

\author{
Hyung Hwa Jeong, Joon Pio Hong, Hyun Suk Suh \\ Department of Plastic and Reconstructive Surgery, Asan Medical Center, University of Ulsan College of Medicine, Seoul, Korea
}

Elevating thin flaps has long been a goal of reconstructive surgeons. Thin flaps have numerous advantages in reconstruction. In this study, we present a surgical method for elevating a thin flap and demonstrate the safety of the procedure. A retrospective review was performed of the electronic medical records of patients who underwent thin flap elevation for lower extremity reconstruction from April 2016 to September 2016 at the Department of Plastic Surgery of Asan Medical Center. All flaps included in this study were elevated above the superficial fascia. A total of 15 superficial circumflex iliac artery free flaps and 13 anterolateral thigh free flaps were enrolled in the study. The total complication rate was $17.56 \%(n=5)$, with total loss of the flap in one patient (3.57\%) and partial necrosis of the flap in four patients (14.28\%). No wound dehiscence or graft loss at the donor wound took place. Elevation above the superficial fascia is not inferior in terms of flap necrosis risk and is superior for reducing donor site morbidity. In addition to its safety, it yields good aesthetic results.

\author{
Correspondence: Hyun Suk Suh \\ Department of Plastic and \\ Reconstructive Surgery, Asan Medical \\ Center, University of Ulsan College of \\ Medicine, 88 Olympic-ro 43-gil, \\ Songpa-gu, Seoul 05505, Korea \\ Tel: $+82-2-3010-3600$ \\ Fax: +82-2-476-7471 \\ E-mail: hyunsuk.suh@amc.seoul.kr
}

Keywords Lower extremity / Free tissue flaps / Subcutaneous tissue

Received: 19 Jul $2017 \bullet$ Revised: 20 Jun $2018 \bullet$ Accepted: 26 Jun 2018

pISSN: 2234-6163 • elSSN: 2234-6171 • https://doi.org/10.5999/aps.2017.01529 • Arch Plast Surg 2018;45:304-313

\section{INTRODUCTION}

Elevating thin flaps has long been a goal of reconstructive surgeons. Thin flaps have numerous advantages in reconstruction, and are required in aesthetically significant areas, such as in facial and hand reconstruction. In addition to their aesthetic importance, thin flaps are frequently required due to functional considerations. In head and neck reconstruction, bulky flaps may interfere with smooth swallowing and cause airway obstruction [1]. For those reasons, many efforts have been made by reconstructive surgeons to achieve thin and durable flaps. In this study, we present a surgical method for elevating thin flaps and demonstrate the safety of the procedure.

\section{PATIENTS AND METHODS}

A retrospective review of electronic medical records at the Department of Plastic Surgery, Asan Medical Center was done. Patients who underwent thin flap elevation on the lower extremity from April 2016 to September 2016 were selected. The operations were performed by two surgeons. For each patient, individual inpatient and outpatient records were reviewed, including the operative record. The data included demographic information, preoperative and operative characteristics, and complications related to the donor site and surgical wound. The operating time was measured from the incision to the end of wound closure, including recipient vessel preparation and debridement. The demographic characteristics of the patients are documented in Table 1. 


\begin{tabular}{|lc|}
\hline Table 1. Clinical and wound characteristics $(\boldsymbol{n}=\mathbf{2 8})$ \\
\hline Characteristic & No. \\
\hline Flap & \\
SCIP & 15 \\
ALT & 13 \\
Sex & \\
Male & 16 \\
Female & 12 \\
Diabetes mellitus & 8 \\
Peripheral vascular disease & 5 \\
Hypertension or coronary arterial disease & 9 \\
Current smoking & 0 \\
Etiology of the defect & \\
Trauma & 12 \\
Malignancy & 6 \\
Diabetes mellitus foot & 5 \\
Chronic osteomyelitis & 2 \\
Others & 3 \\
Defect location & \\
Foot & 19 \\
Leg & 8 \\
Thigh & 1 \\
\hline SCIP, superficial circumflex iliac artery perforator flap; ALT, anterolateral thigh free \\
flap. \\
\hline
\end{tabular}

\section{SURGICAL PROCEDURE}

Preoperatively, perforator mapping was performed at the planning stage. The mapping was conducted using computed tomography (CT) angiography and color Doppler imaging. First, using CT angiography, we roughly located the perforator penetrating the deep fascia and characterized the overall vascular status of the extremity. Preoperative markings of perforators were made based on the anatomical landmarks described by Celik et al. [2] for anterolateral thigh (ALT) free flaps, and Hong et al. [3] for superficial circumflex iliac perforator (SCIP) flaps. Using $\mathrm{CT}$ angiography, we were able to note the level of perforator penetration. Then, we utilized color Doppler imaging to identify the exact shape of the perforator and its running direction in the adipose layer. Flap design was performed on the basis of the preoperative perforator mapping.

Flap elevation was performed as Hong et al. [4] previously described for superficial fascia elevation. Medial and lateral incisions were made at once around the margin of the flap. Freestyle flap elevation was performed under loupe magnification from one of the incisions until the perforators were reached. The superficial fascia is located between the large deep fat lobules and the smaller superficial fat lobules. The size of the fat lobules changes just after passing a very thin fascia-like structure. To elevate the flap at the right layer, adequate tension using a self-retracting hook or manual retraction by an assistant is necessary.

\section{Fig. 1. Thickness of SCIP flap with thin elevation}

The elevated flap can be as thin as $5 \mathrm{~mm}$. SCIP, superficial circumflex iliac artery perforator.

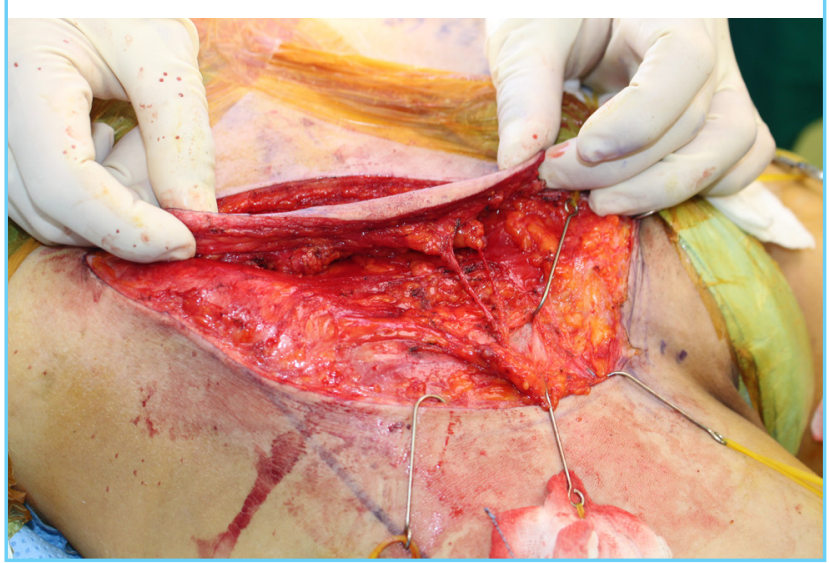

The flap was elevated above the superficial fascia. Once the perforators were found during the process of elevating the flap above the superficial fascia, the biggest perforator was usually saved, and dissection was continued into the deep fascia and muscle until an adequate length of the pedicle was achieved. If the flap was large or there was no large $(>0.2-0.3 \mathrm{~mm}$ ) perforator above the superficial fat layer, multiple perforators were preserved, dissected, and skeletonized to the deep fat layer to ensure that the flap would receive sufficient blood supply. Flaps as thin as $5 \mathrm{~mm}$ could also be elevated over the superficial fascia (Fig. 1).

\section{RESULTS}

Between April 2016 to September 2016, the patient group included 15 superficial circumflex iliac artery free flaps and 13 ALT free flaps. The average age of the patients was 48.33 years (range, 5-83 years). All flaps were successfully elevated over the superficial fascia layer with at least a single viable perforator ( $\mathrm{Ta}-$ ble 1). The etiology of the defect was trauma in 12 cases, malignancy in six cases, diabetic foot in five cases, and chronic osteomyelitis in two cases. Other etiologies included gout and unstable scars. The patients' comorbidities included diabetes $(n=8)$, peripheral vessel disease $(n=5)$, and hypertension or coronary arterial disease $(n=11)$. Half of the patients had at least one comorbidity.

The operative characteristics are shown in Table 2. The average size of the ALT flaps was $16.04 \pm 7.46 \mathrm{~cm}$ in length and $5.22 \pm 2.45 \mathrm{~cm}$ in width (range, $10 \times 4$ to $28 \times 13 \mathrm{~cm}$ ). The average size of the SCIP flaps was $15.51 \pm 4.93 \mathrm{~cm}$ in length and $7.18 \pm 2.33 \mathrm{~cm}$ in width (range, $7 \times 5$ to $22 \times 11 \mathrm{~cm}$ ). Most of the arterial anastomoses were performed in an end-to-side manner $(n=19)$. Primary closure of the donor site was possible in the 


\section{Table 2. Operative data $(n=28)$}

\begin{tabular}{lc}
\hline Characteristic & No. \\
\hline Flap & \\
SCIP & 15 \\
ALT & 13 \\
Average flap size (cm) & \\
ALT & \\
$\quad$ Length & $16.04 \pm 7.46$ \\
$\quad$ Width & $5.22 \pm 2.45$ \\
SCIP & \\
$\quad$ Length & $15.51 \pm 4.93$ \\
$\quad$ Width & $7.18 \pm 2.33$ \\
Average total operation time (min) & \\
SCIP & 265.36 \\
ALT & 271.08 \\
Perforators & \\
1 & 22 \\
2 & 4 \\
3 & 2 \\
Donor site closure & \\
Primary & \\
Skin graft & \\
Arterial anastomosis type & \\
End-to-side & \\
End-to-end & \\
Flow through & \\
Venous anastomosis & 10 \\
1 & \\
2 & \\
3 & \\
Values are presented number or mean \pm standard deviation. & \\
SCIP, superficial circumflex iliac artery perforator flap; ALT, anterolateral thigh free \\
flap. \\
a)All donor sites of SCIP flaps were primarily closed. \\
$\quad$ \\
\end{tabular}

majority of the patients; the donor sites of the SCIP free flaps were all closed primarily, while a split-thickness skin graft was used in nine ALT free flap cases.

The total complication rate was $17.56 \%(n=5)$, with total loss of the flap in one patient (3.57\%) and partial necrosis of the flap in four patients (14.28\%). In one case with a complication, a major revision requiring reoperation was performed, including flap take-down and elevation of a re-do flap. Minor revision with an additional skin graft was performed in three cases of partial necrosis. After revision surgery, all flaps survived. No wound dehiscence or graft loss at the donor wound was found. A secondary thinning procedure was performed for one ALT flap and one SCIP flap. The overall characteristics of the complications are presented in Table 3.

\section{CASE REPORTS}

\section{Case 1}

A 75-year-old man was referred to the Department of Plastic

\begin{tabular}{|c|c|}
\hline Characteristic & No. $(\%)$ \\
\hline \multicolumn{2}{|l|}{ Total loss } \\
\hline ALT & 0 \\
\hline SCIP & $1(3.57)$ \\
\hline Total & $1(3.57)$ \\
\hline \multicolumn{2}{|l|}{ Partial necrosis } \\
\hline ALT & $2(7.14)$ \\
\hline SCIP & $2(7.14)$ \\
\hline Total & $4(14.28)$ \\
\hline \multicolumn{2}{|l|}{ Major revision } \\
\hline ALT & 0 \\
\hline SCIP & $1(3.57)$ \\
\hline Total & $1(3.57)$ \\
\hline \multicolumn{2}{|l|}{ Minor revision } \\
\hline ALT & 0 \\
\hline SCIP & $3(10.71)$ \\
\hline Total & $3(10.71)$ \\
\hline \multicolumn{2}{|l|}{ Donor site morbidity } \\
\hline ALT & 0 \\
\hline SCIP & 0 \\
\hline Total & 0 \\
\hline \multicolumn{2}{|c|}{ Secondary thinning procedure } \\
\hline ALT & $1(3.57)$ \\
\hline SCIP & $1(3.57)$ \\
\hline Total & $2(7.14)$ \\
\hline
\end{tabular}

and Reconstructive Surgery with a diagnosis of open fracture at the left fibula and medial malleolus with a diffuse soft tissue defect in the ipsilateral pretibial area (Fig. 2A). In cooperation with an orthopedic surgery team, bone fixation and soft tissue coverage with a free flap were planned. An external fixator was applied by the orthopedic surgeons to fix the bone. After debridement of the devitalized skin, an ALT flap measuring $28 \times 13 \mathrm{~cm}$ at the ipsilateral thigh was designed. The flap was elevated above the superficial fascia (Fig. 2B and C). The pedicle anastomosis was performed in an end-to-side manner to the posterior tibial artery and in an end-to-end manner to the vena comitans. At 8 months postoperatively, the patient showed a good and thin contour. The donor site also healed well, without any complications (Fig. 2D).

\section{Case 2}

A 30-year-old woman presented to the clinic of the Department of Plastic and Reconstructive Surgery with a chief complaint of right first toe skin necrosis accompanied by bone exposure after open reduction and internal fixation by a local orthopedic surgeon due to a crushing injury. After debridement, coverage with a flap was planned (Fig. 3A). A SCIP free flap measuring $10 \times 4$ $\mathrm{cm}$ was designed at the ipsilateral groin. Flap elevation was per- 


\section{Fig. 2. Pretibial reconstruction with thin ALT free flap}

(A) Preoperative findings of the defect. (B) Design of the anterolateral thigh (ALT) free flap. (C) Harvested free flap that was elevated above the superficial fascia. (D) A follow-up photograph taken 8 months postoperatively.
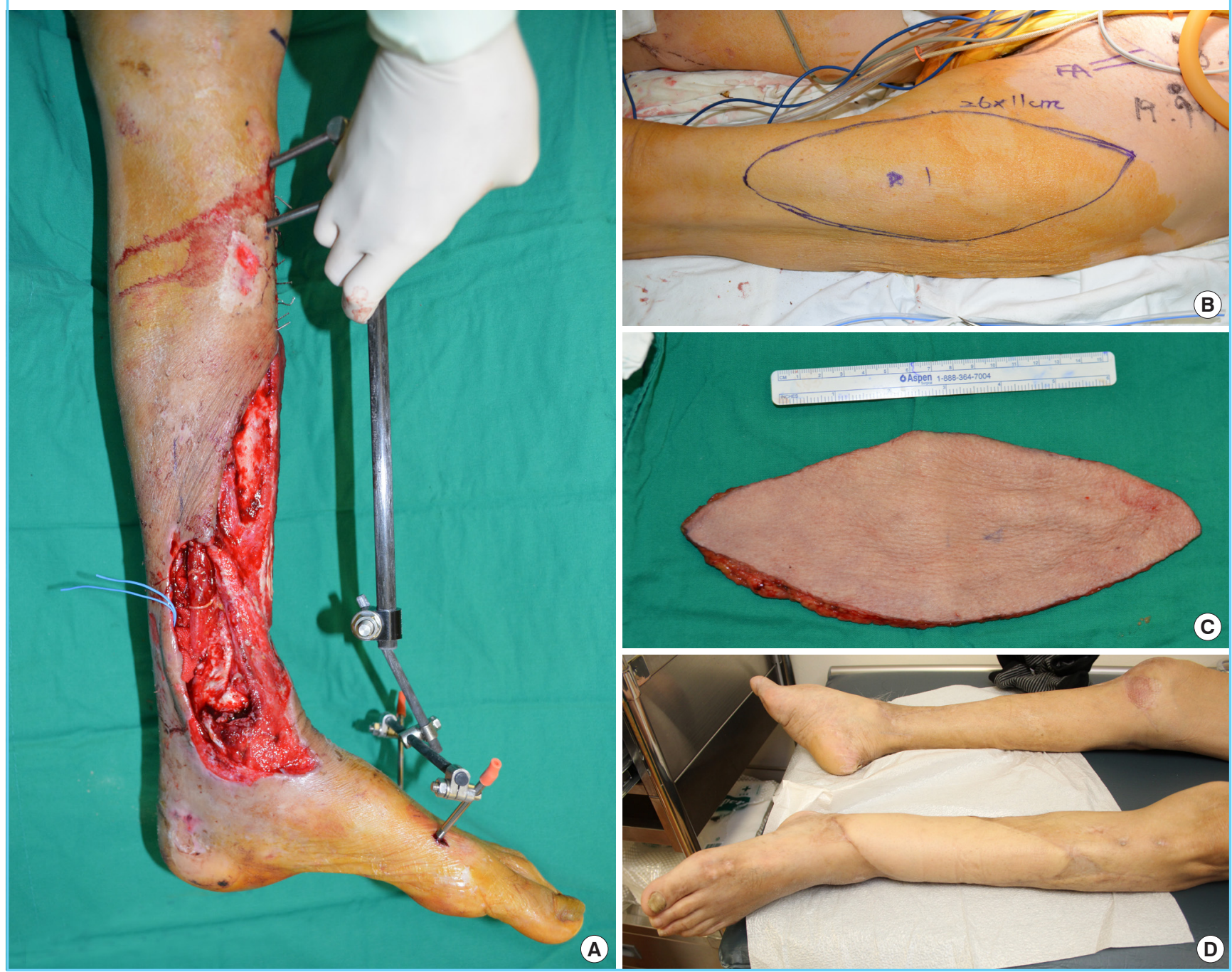

formed above the superficial fascia (Fig. 3B and C). Vascular anastomosis was performed in a flow-through manner for arterial anastomosis to maintain the distal flow. End-to-end anastomoses for two venae comitantes was performed. The donor site was closed primarily. Fig. 3D shows the immediate postoperative findings. The patient was discharged on postoperative day 12 , without major complications. At 6 months postoperatively, the contour of the flap was thin and fit the dorsum contour. The donor site healed completely, without complications (Fig. 3E-G).

\section{Case 3}

A 17-year-old woman who was a modern dancer by occupation was referred from the emergency trauma team to the Department of Plastic and Reconstructive Surgery because of a degloving injury of the right foot dorsum due to a pedestrian traffic ac- cident. She had already undergone external fixation for a digital fracture. The orthopedic team performed debridement of the devitalized bone and amputated the first to third proximal phalanx and the fifth mid-phalanx. After dressing with negative pressure wound therapy, dorsum skin coverage with a flap was planned (Fig. 4A and B). A SCIP free flap measuring $22 \times 11 \mathrm{~cm}$ was designed at the ipsilateral groin. Flap elevation was performed above the superficial fascia, preserving the deep fat layer. The patient had low body weight, and the deep fat was scanty (Fig. 4B and C). Microvascular anastomosis was performed on the dorsalis pedis artery in an end-to-side fashion and two venae comitantes were anastomosed in an end-to-end manner. The donor site was closed primarily. At 1 year postoperatively, the contour was good, without bulging. She resumed modern dancing (Fig. 4D). 

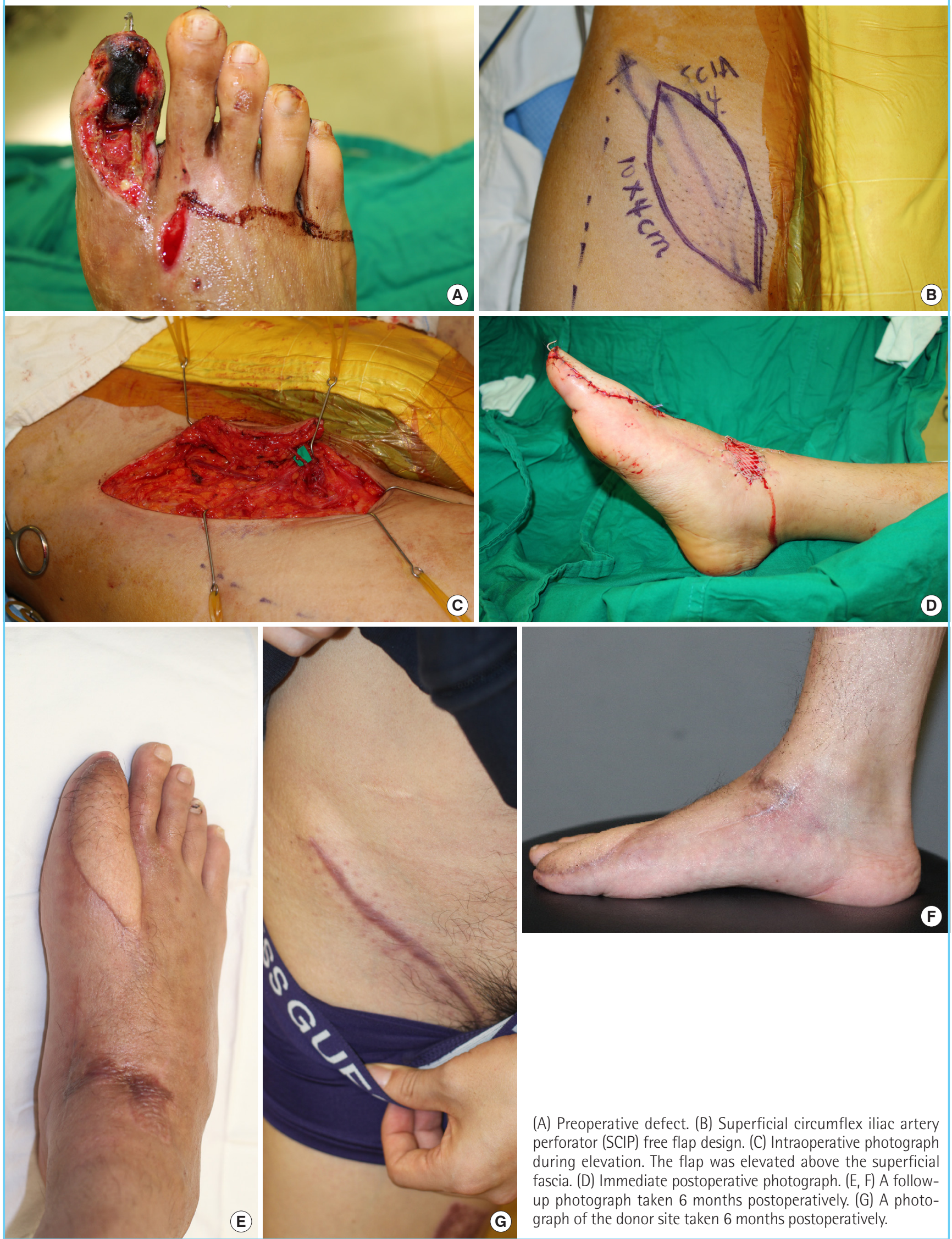

(A) Preoperative defect. (B) Superficial circumflex iliac artery perforator (SCIP) free flap design. (C) Intraoperative photograph during elevation. The flap was elevated above the superficial fascia. (D) Immediate postoperative photograph. (E, F) A followup photograph taken 6 months postoperatively. (G) A photograph of the donor site taken 6 months postoperatively. 


\section{Fig. 4. Forefoot reconstruction with thin SCIP free flap}

(A, B) Preoperative photograph of the defect. (C) Harvested superficial circumflex iliac artery perforator (SCIP) free flap. (D) Photograph taken during elevation. The flap was elevated above the superficial fascia. The deep fat was scanty because of the patient's low body weight. A follow-up photograph taken 1 year postoperatively.
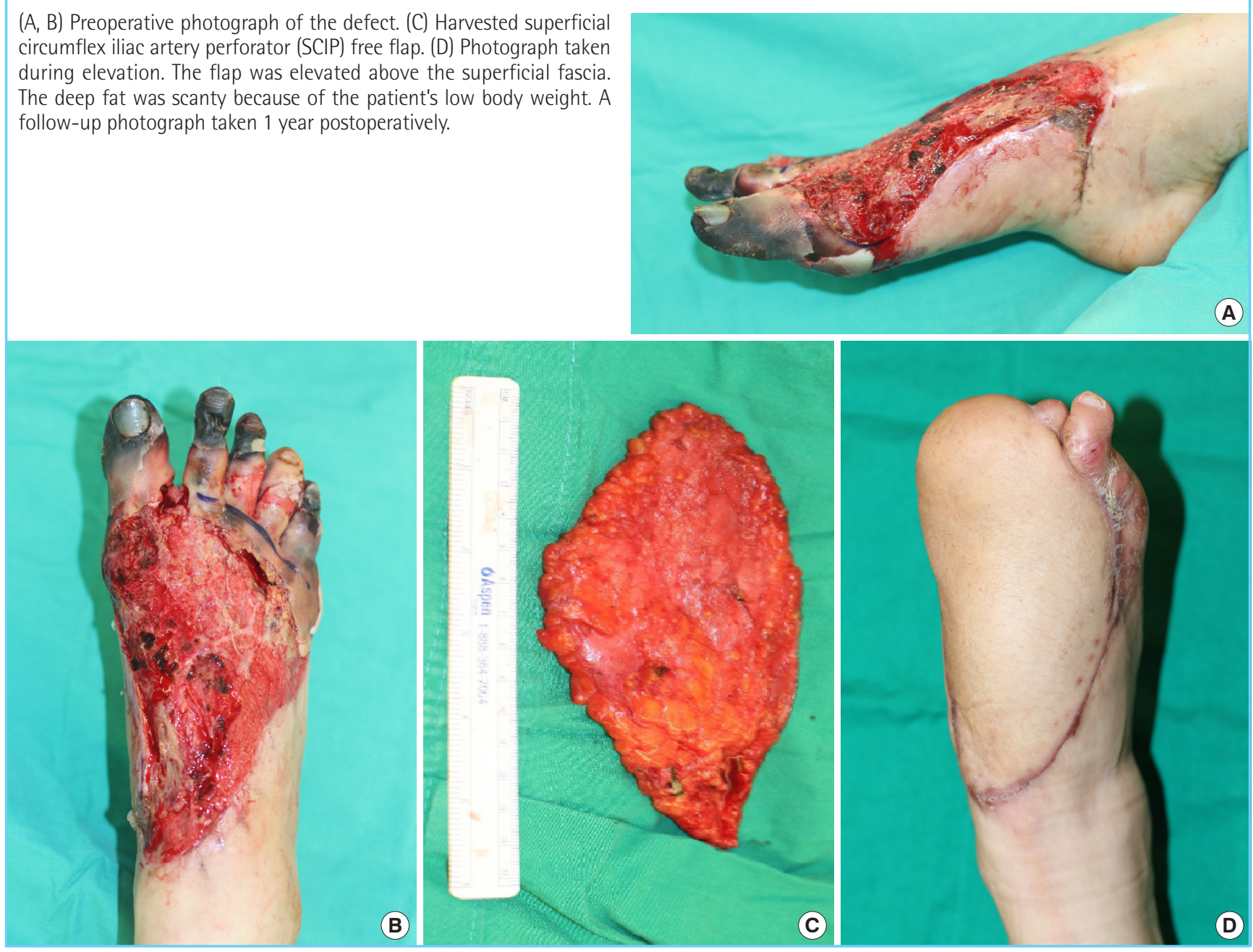

\section{Case 4}

A 67-year-old woman presented to the clinic with a chief complaint of skin necrosis in the right heel area after open reduction and internal fixation at a local orthopedic clinic because of an ankle fracture (Fig. 5A). The patient diabetes and hypertension as underlying diseases. On preoperative $\mathrm{CT}$ angiography, occlusion of the right popliteal artery was suspected, but distal flow was maintained. We decided to perform soft tissue coverage after debridement. After debridement, a defect measuring $12 \times 6$ $\mathrm{cm}$ remained. A superficial circumflex iliac artery free flap measuring $15 \times 6 \mathrm{~cm}$ was elevated from the contralateral groin, preserving the superficial fascia (Fig. 5B). Arterial anastomosis was performed in an end-to-end manner to the muscular perforator of the peroneal artery. Venous anastomosis was performed to the superficial vein in an end-to-end manner (Fig. 5C). At 4 days postoperatively, distal congestion started. After demarcation, we debrided the overlying devitalized skin and noted that the subcutaneous layer was viable (Fig. 5D). Negative pressure wound therapy was applied for 5 months. With several rounds of debridement and staged closure, the heel defect was closed (Fig. 5E).

\section{DISCUSSION}

Elevating thin flaps has long been a goal of reconstructive surgeons. Thin flaps have numerous advantages for reconstruction, and there is no doubt that they are superior to thick flaps in terms of meticulously mimicking natural contours. Thin flaps are required for aesthetically significant areas, such as in facial and hand reconstruction. In order to cover the surface with a pliable and thin flap in an aesthetically pleasing manner, fascial free flaps with a skin graft were traditionally used $[5,6]$. In addition to aesthetic considerations, thin flaps are frequently required for functional reasons. In head and neck reconstruction, protection after resurfacing is the main goal of surgery. Thus, establishing a well-vascularized tissue bed that can tolerate future 


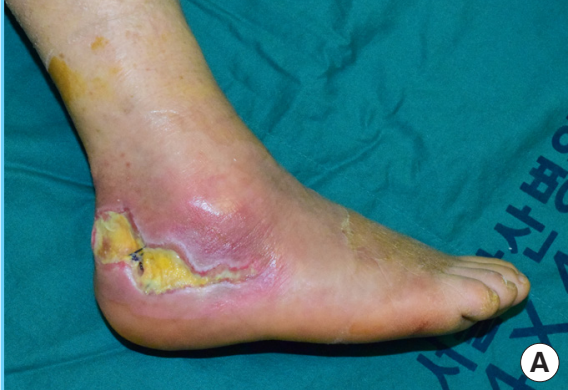

(A) Preoperative photograph. (B) Harvested superficial circumflex iliac artery perforator (SCIP) free flap. (C) Immediate postoperative photograph. (D) A photograph taken after debridement of the partially necrotized portion of the flap. Negative pressure wound therapy was applied for 5 months. (E) A photograph of the totally healed flap. SCIP, superficial circumflex iliac artery perforator.
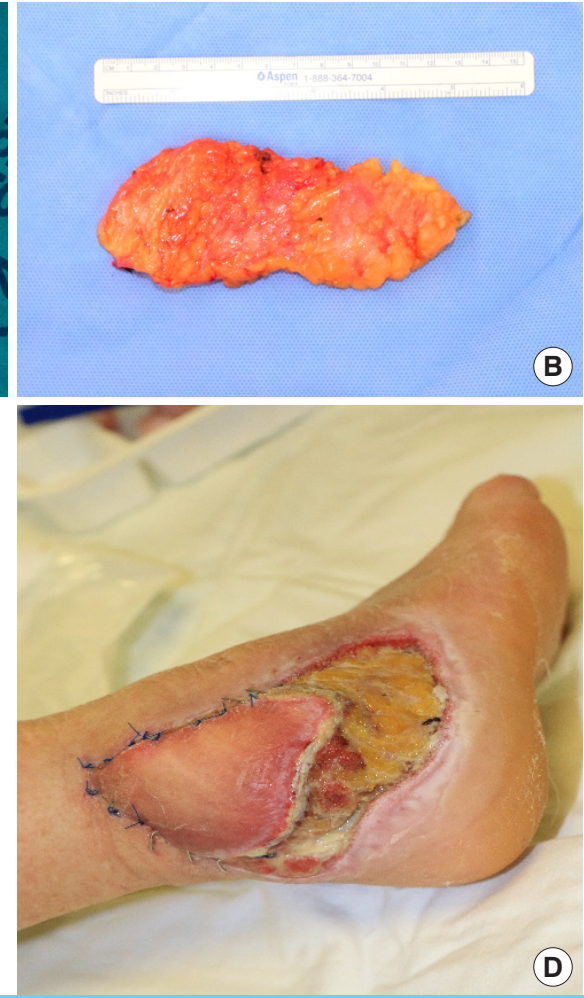

(D)
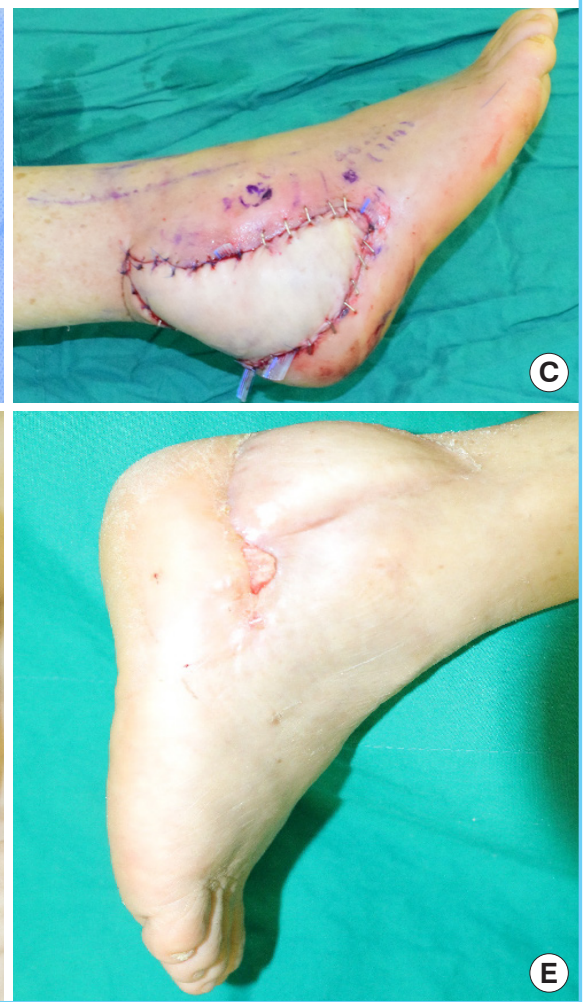

(E)

radiation therapy, thereby preventing fistula formation, is critical in reconstructive operations [1]. However, bulky flaps in the head, hand, and neck region may cause critical problems. Bulky flaps may interfere with smooth swallowing and cause airway obstruction [1]. In extremity reconstruction, reconstructive surgeons may frequently experience mechanical injuries in reconstructed tissue because of excessive bulk, which may cause shearing or abnormal contact. Moreover, bulky flaps on the foot dorsum or toes can make it difficult for patients to wear shoes, potentially affecting foot function [7].

For those reasons, reconstructive surgeons have made many efforts to achieve thin and durable flaps. Debulking procedures have been performed as a classical method to control the thickness of the flap. Direct excision of the fatty layer or liposuction is widely used as a debulking method. Secondary debulking procedures after lower extremity flap coverage have been reported to be performed in $17 \%$ to $52 \%$ of cases [7-9]. In face reconstruction, complicated contour and color matching is also difficult to obtain, meaning that additional revisional procedures are often required in order to achieve an acceptable contour [10]. However, secondary debulking procedures have some pitfalls. First, they cause additional costs, and are not covered by the national health insurance system in South Korea. Circulation issues are another problem of debulking procedures. Direct exci- sion largely depends on the dermal-subdermal plexus, and several stages are required to ensure the viability of the skin [11].

In order to control the thickness of the flap, we advocate suprafacial elevation. As described above, the goal of this technique is to elevate the flap over the superficial fascia, preserving not only the deep fascia, but also the deep fat layer below the superficial fascia. By elevating the flap in a way that preserves the superficial fascia, we were able to achieve a very thin flap and increase pliability at the same time.

Combined with super-microsurgery techniques, we can maximize the utility of supra-superficial fascial flaps. We perform perforator-to-perforator techniques unless a long pedicle is required, to minimize recipient vessel morbidity by preserving the large vessel to the distal area. Not using the main vessel as a donor vessel is advantageous in cases of extreme calcification and atherosclerosis. Theoretically, in such cases, the perforator level is less affected than large vessels. When elevating an ALT free flap, the lateral circumflex femoral artery, including its descending branch is not atherosclerosis-resistant [12]. As a result, in severe cases of atherosclerosis, especially in diabetic foot patients, we cannot guarantee the patency of the donor vessel, even if we use the larger branch as a donor.

Theoretically, it was considered that not including the fascia would decrease flap perfusion. However, partial necrosis does 


\section{Table 4. Predictors of partial flap necrosis}

\begin{tabular}{|c|c|c|c|c|}
\hline Characteristics & $\begin{array}{c}\text { No } \\
\text { necrosis } \\
(n=23)\end{array}$ & $\begin{array}{c}\text { Partial } \\
\text { necrosis } \\
(n=4)\end{array}$ & P-value & $\begin{array}{l}\text { Odds } \\
\text { ratio }\end{array}$ \\
\hline Peripheral vascular disease & 2 & 3 & $0.013^{\mathrm{a})}$ & 31.5 \\
\hline Diabetes & 5 & 1 & 0.731 & 0.944 \\
\hline Hypertension/coronary arterial disease & 7 & 3 & 0.093 & 8.5 \\
\hline Over 2 comorbidities & 4 & 2 & 0.204 & 4.75 \\
\hline
\end{tabular}

a)Statistically significant. Odds ratio, Pearson chi-square test, Fisher exact test.

not generally occur in flaps after suprafascial elevation. The interconnections between direct and indirect linking vessels create interperforator flow [13]. Suprafascial flap viability is explained by the perforasome theory [14]. A supra-superficial fascial flap might not include a direct linking vessel located at the subcutaneous layer and suprafascial plexus. However, as we can also conclude from our results, partial necrosis of supra-superficial fascial flaps due to impaired circulation does not occur to a greater extent than in formal suprafascial flaps and fasciocutaneous flaps. Large thin flaps can survive without direct linking vessels $[4,15,16]$.

One case of total loss of the flap in our study was reported. However, we do not think that this result indicates an inherent risk associated with technique of flap elevation over the superficial fascia. The reason for necrosis was a delayed infection postoperatively. The patient had flap coverage because of an unstable scar with underlying chronic osteomyelitis, and had diabetes and poor nutritional status. Preoperative nutritional status has been described as an accurate predictor of postoperative outcomes [17]. Prealbumin is a visceral protein that is commonly used to evaluate nutritional status [18], and the patient had a low prealbumin level at admission.

The occurrence of partial necrosis is another issue. Predicting whether a flap will completely survive prior to surgery is of vital interest to reconstructive surgeons. In our results, three of the patients who experienced partial necrosis showed peripheral vascular disease on preoperative CT angiography. Suh et al. [19] previously reported peripheral arterial disease to be a factor significantly related to flap loss. All patients with partial necrosis of the flap had at least one underlying comorbidity, and peripheral vascular disease was significantly related to partial necrosis (odds ratio $=31.5, \mathrm{P}=0.013$ ). We presume that the comorbidities contributed to the flap necrosis, not the surgical technique of flap elevation (Table 4). Furthermore, no cases of partial necrosis resulted in bone exposure or implant exposure requiring additional flap coverage. When planning a reconstruction with a superficial fascia-preserving flap, some modalities can be used to anticipate the proportion of necrosis and to prepare or modi- fy the plan. Indocyanine green and fluorescence images can precisely predict tissue viability [20]. With precise planning using $\mathrm{CT}$ angiography and color Doppler imaging, we are confident that surgeons can minimize flap morbidity.

A defatting procedure during flap elevation is another option for controlling flap thickness without a secondary revisionary procedure. However, immediate debulking during flap elevation cannot be done while distinguishing the exact anatomical plane, which can result in subdermal or subcutaneous vessel connection $[21,22]$. Immediate debulking is more dangerous than identifying and elevating the exact plane of the superficial fascia, preserving the subdermal plexus. As our study shows, elevating flaps thinner than $5 \mathrm{~mm}$ above the superficial fascia can reduce the defatting procedure rate, thereby avoiding the risk of damaging the subdermal plexus.

Delayed wound healing at the donor site is a significant issue in achieving an ultimately successful reconstruction. Many factors can impede wound healing. In the elevation of radial forearm flaps, by preserving the deep fascia of the forearm, less tendon exposure was reported [23]. In our study, an extremely low percentage of donor site graft loss was noted. We presume that by leaving the deep fat layer at the donor site, the viability of the skin graft was maximized.

Sensory impairment at the donor site after free flap elevation is one of the most frequently reported morbidities, and abnormal sensation at the donor site is among the most common subjective complaints from patients $[14,24]$. Elevating ALT free flaps over the superficial fascia was reported to lead to fewer cases of abnormal sensation than in cases of subfascial ALT free flaps due to preservation of the fascial layer [14]. The medial branch of the lateral cutaneous femoral nerve is often sacrificed during ALT flap harvesting [25]. Moreover, it is still possible for an anterolateral flap elevated over the superficial fascia to utilize the sensory nerve for a sensate flap, which is an inherent advantage of the ALT free flap $[16,26]$. The sensory nerve utilized in sensate ALT free flaps is the lateral femoral cutaneous nerve, which runs at the deep fat layer [14]. Surgeons can isolate and dissect the nerve during supra-superficial fascial elevation, even if the nerve is located beneath the superficial fascia. Seth and Iorio [16] reported using a sensitized ALT free flap that was elevated above the superficial fascia.

Groin flaps lost their popularity because of frequent donor site morbidity. They were introduced as a good flap option with well-concealed donor site scar, but lost favor because of their bulky nature and frequent donor site-related complications, such as wound dehiscence and lymphorrhea [3]. Traditional groin flaps included parts of the deep fascial structure. Because the lymphatic system is located in a deep fat layer, which is un- 
derneath the superficial fascia, including the tissues beneath the superficial fascia results in lymphorrhea and delayed wound healing. In a modified version of SCIP flap elevation, in which the elevation is performed above the superficial fascia, the donor site-related problems were reduced dramatically [3]. There were no cases of wound dehiscence or delayed healing in our group of SCIP cases, precisely corresponding with previous reports.

The elevation time of suprafascial thin flaps compared to flaps elevated in the subfascial plane is controversial. Chen et al. [14] reported no significant difference in elevation time between suprafascial and subfascial ALT flaps. Some studies reported that thinning flaps or elevating thin flaps was more time-consuming [27]. Of course, elevating a free flap while preserving the superficial fascia has a steep learning curve. However, a study by Seth and Iorio [16] reported a shorter operating time in superficial fascia-preserving elevation than in formal suprafascial flap elevation for ALT perforator flaps. In our results, the operating time in both the ALT and SCIP groups was comparable with previous studies [16].

\section{CONCLUSION}

Elevation of the flap over the superficial fascia represents an important refinement to the traditional fasciocutaneous flap. We argue that elevation above the superficial fascia is not inferior in terms of flap necrosis risk and is superior in terms of reducing donor site morbidity.

\section{NOTES}

\section{Conflict of interest}

No potential conflict of interest relevant to this article was reported.

\section{Ethical approval}

The study was approved by the Asan Institute for Life Sciences, Asan Medical Center (IRB No. S2018-0915-0001) and performed in accordance with the principles of the Declaration of Helsinki. Written informed consents were obtained.

\section{Patient consent}

The patients provided written informed consent for the publication and the use of their images.

\section{REFERENCES}

1. Garg RK, Poore SO, Wieland AM, et al. Elective free flap re- vision in the head and neck cancer patient: indications and outcomes. Microsurgery 2015;35:591-5.

2. Celik N, Wei FC, Lin CH, et al. Technique and strategy in anterolateral thigh perforator flap surgery, based on an analysis of 15 complete and partial failures in 439 cases. Plast Reconstr Surg 2002;109:2211-6.

3. Hong JP, Sun SH, Ben-Nakhi M. Modified superficial circumflex iliac artery perforator flap and supermicrosurgery technique for lower extremity reconstruction: a new approach for moderate-sized defects. Ann Plast Surg 2013;71: 380-3.

4. Hong JP, Choi DH, Suh H, et al. A new plane of elevation: the superficial fascial plane for perforator flap elevation. J Reconstr Microsurg 2014;30:491-6.

5. Lee MG, Kim JS, Lee DC, et al. Fascial free flap for reconstruction of the dorsolateral hand and digits: the advantage of a thin contour. Arch Plast Surg 2016;43:551-8.

6. Baujat B, Struk S, Lesnik M, et al. Fascia temporalis free flap for cricotracheal reconstruction: a novel approach. Ann Thorac Surg 2017;104:1040-6.

7. Lee KT, Park SJ, Mun GH. Reconstruction outcomes of oncologic foot defect using well-contoured free perforator flaps. Ann Surg Oncol 2017;24:2404-12.

8. Kuran I, Turgut G, Bas L, et al. Comparison between sensitive and nonsensitive free flaps in reconstruction of the heel and plantar area. Plast Reconstr Surg 2000;105:574-80.

9. Hollenbeck ST, Woo S, Komatsu I, et al. Longitudinal outcomes and application of the subunit principle to 165 foot and ankle free tissue transfers. Plast Reconstr Surg 2010; 125:924-34.

10. Choi DH, Goh T, Cho JY, et al. Thin superficial circumflex iliac artery perforator flap and supermicrosurgery technique for face reconstruction.J Craniofac Surg 2014;25:2130-3.

11. Clemens MW, Colen LB, Attinger CE. Foot reconstruction. In: Neligan PC, Song DH, editors. Plastic surgery. London: Elsevier Saunders; 2013. p. 189-218.

12. Burusapat C, Nanasilp T, Kunaphensaeng P, et al. Effect of atherosclerosis on the lateral circumflex femoral artery and its descending branch: comparative study to nonatherosclerotic risk. Plast Reconstr Surg Glob Open 2016;4:e856.

13. Saint-Cyr M, Wong C, Schaverien M, et al. The perforasome theory: vascular anatomy and clinical implications. Plast Reconstr Surg 2009;124:1529-44.

14. Chen YC, Scaglioni MF, Carrillo Jimenez LE, et al. Suprafascial anterolateral thigh flap harvest: a better way to minimize donor-site morbidity in head and neck reconstruction. Plast Reconstr Surg 2016;138:689-98.

15. Hong JP, Chung IW. The superficial fascia as a new plane of 
elevation for anterolateral thigh flaps. Ann Plast Surg 2013; 70:192-5.

16. Seth AK, Iorio ML. Super-thin and suprafascial anterolateral thigh perforator flaps for extremity reconstruction. J Reconstr Microsurg 2017;33:466-73.

17. Khuri SF, Daley J, Henderson W, et al. Risk adjustment of the postoperative mortality rate for the comparative assessment of the quality of surgical care: results of the National Veterans Affairs Surgical Risk Study. J Am Coll Surg 1997; 185:315-27.

18. Devoto G, Gallo F, Marchello C, et al. Prealbumin serum concentrations as a useful tool in the assessment of malnutrition in hospitalized patients. Clin Chem 2006;52:2281-5.

19. Suh HS, Oh TS, Lee HS, et al. A new approach for reconstruction of diabetic foot wounds using the angiosome and supermicrosurgery concept. Plast Reconstr Surg 2016;138: 702e-709e.

20. Wada H, Vargas CR, Angelo J, et al. Accurate prediction of tissue viability at postoperative day 7 using only two intraoperative subsecond near-infrared fluorescence images. Plast Reconstr Surg 2017;139:354-63.

21. Sharabi SE, Hatef DA, Koshy JC, et al. Is primary thinning of the anterolateral thigh flap recommended? Ann Plast
Surg 2010;65:555-9.

22. Sun G, Lu M, Tang E, et al. Clinical application of free anterolateral thigh flap in the reconstruction of intraoral defects. Oral Surg Oral Med Oral Pathol Oral Radiol Endod 2011;112:34-41.

23. Shonka DC Jr, Kohli NV, Milam BM, et al. Suprafascial harvest of the radial forearm free flap decreases the risk of postoperative tendon exposure. Ann Otol Rhinol Laryngol 2017;126:224-8.

24. Fischer S, Klinkenberg M, Behr B, et al. Comparison of donor-site morbidity and satisfaction between anterolateral thigh and parascapular free flaps in the same patient. J Reconstr Microsurg 2013;29:537-44.

25. Ribuffo D, Cigna E, Gargano F, et al. The innervated anterolateral thigh flap: anatomical study and clinical implications. Plast Reconstr Surg 2005;115:464-70.

26. Ali RS, Bluebond-Langner R, Rodriguez ED, et al. The versatility of the anterolateral thigh flap. Plast Reconstr Surg 2009;124(6 Suppl):e395-407.

27. Adani R, Tarallo L, Marcoccio I, et al. Hand reconstruction using the thin anterolateral thigh flap. Plast Reconstr Surg 2005;116:467-73. 